Notfallanalgesie

\section{Nierenkolik: NSAR statt Opioid?}

— Die gängige Praxis, bei Nierenkolik ein Opioid i.v. zu geben, ist offenbar nicht optimal. Eine wirksamere Schmerzlinderung wurde in einer Studie mit der I. m.-Applikation eines NSAR erreicht. Laut der doppelblinden und randomisiert-kontrollierten Studie könnte für einen Großteil der Patienten, die mit Nierenkolik eine Notaufnahme aufsuchen, ein NSAR i.m. das Analgetikum der Wahl sein [Pathan et al. Lancet 2016; 387 (10032): 1999-2007]. An der Studie waren 1.644 Notaufnahmepatienten mit Verdacht auf Nierenkolik beteiligt. Die Schmerzen hatten sie auf einer numerischen Skala (NRS) von 1- 10 im Median mit 8 Punkten bewertet. 547 Teilnehmer erhielten Diclofenac (75 $\mathrm{mg} / 3 \mathrm{ml}$ i. m.), 548 Paracetamol $(1 \mathrm{~g} / 100 \mathrm{ml}$ i. v.) und 549 Morphin $(0,1 \mathrm{mg} / \mathrm{kg} \mathrm{KG}$ i. v.). Um die Studientherapie verblinden zu können, bekamen alle Patienten drei Injektionen, eine mit Wirkstoff und zwei mit Placebo.

Eine mindestens $50 \%$ ige Schmerzreduktion nach 30 Minuten, das primäre Studienziel, wurde mit Diclofenac von $68 \%$, mit Paracetamol von $66 \%$ und mit Morphin von $61 \%$ der Patienten erreicht. Der Unterschied von Diclofenac, aber nicht der von Paracetamol zu Morphin war signifikant. Die NSAR-Gruppe benötigte 30 Minuten nach der ersten Analgesie seltener eine RescueAnalgesie als die beiden anderen Gruppen (12\% vs. $20 \%$ bzw. $23 \%$ ). Auch nach $60 \mathrm{Mi}-$ nuten war der Patientenanteil mit persistierenden Schmerzen (NRS > 2) unter Diclofenac am geringsten ( $24 \%$ vs. $30 \%$ bzw. $38 \%$ ). Nach Ansicht der Studienautoren um Sameer Pathan von der Klinik in Hamad "widersprechen die Ergebnisse der klinischen Glaubenslehre, wonach eine intravenöse Analgesie, zumal mit Narkotika, schneller und effektiver ist". Die Resultate hätten daher "tiefgreifende Konsequenzen“ für die Erstversorgung von Nierenkolikpatienten: „NSAR i.m. sollten als Erstlinientherapie betrachtet werden. In diesem Zusammenhang ist allerdings unbedingt zu beachten, dass Patienten mit Niereninsuffizienz, Leberschäden, Asthma oder Schwangerschaft von der Studie ausgeschlossen waren. Sie werden daher von Pathan und Kollegen ausdrücklich von der Empfehlung zur NSARGabe ausgenommen. Dr. Beate Schumacher

Negative Konnotation

\title{
Patienten empfinden palliative Versorgung als Stigma
}

Es gibt keine Diskussion über den Umgang mit Schwerkranken, in der nicht gefordert würde, die Palliativversorgung auszubauen. Doch die Palliativmedizin tut sich noch immer schwer. Ein Grund für dieses zögerliche Vorgehen könnten negative Assoziationen mit dem Begriff Palliativmedizin sein. Jedenfalls lassen dies die Ergebnisse einer Studie vermuten, die eine Arbeitsgruppe um Camilla Zimmermann von der Universität Toronto vorgelegt hat [Zimmermann et al. CMAJ online 2016 Apr 18]. Die Wissenschaftler befragten zwei Gruppen von Patienten, von denen die erste eine onkologische Standardversorgung und die zweite eine palliative Versorgung erhielt. Interviewt wurden auch Betreuer von Patienten der Kontroll- oder der Interventionsgruppe.

Anfangs der Untersuchung verbanden die Patienten und Betreuer in beiden Studienarmen die Palliativversorgung mit Tod, Hoffnungslosigkeit, Abhängigkeit und einer stationären, tröstenden Pflege am Ende des Lebens. Zimmer- mann und Kollegen interessierte die Frage, ob etwaige positive Erfahrungen mit der palliativen Versorgung diese Einstellungen änderten. Eine zweite Befragung vier Monate später ergab, dass die Angehörigen der Interventionsgruppe nun tatsächlich einen etwas anderen Blick auf die Palliation hatten und sie als eine anhaltende Versorgung ansahen, die ihre Lebensqualität verbesserte und keineswegs nur die letzten Tage betraf. Dennoch empfanden sie den Begriff der Palliativversorgung nach wie vor als Stigma. Sie betonten, es sei nötig, das Konzept besser zu erklären und auf einen anderen Namen zu taufen. Dagegen sahen die Teilnehmer der Kontrollgruppe in einer Änderung der Benennung keinen rechten Sinn.

„Ärzte sollten sich darüber im Klaren sein, dass die Form, in der sie Informationen über die Palliativversorgung vermitteln, deren Wahrnehmung beeinflusse - und auch die Entscheidung darüber, solche Pflege anzunehmen", so das Resümee von Zimmermann. Robert Bublak

\section{US-Leitlinien-Update}

\section{Botulinumtoxin auch bei chronischer Migräne empfohlen}

— In klinischen Studien waren die Unterschiede zwischen Placebo und Botulinumtoxin (BTX) relativ gering, dennoch haben sich nun auch US-Neurologen in einem Update zur BTX-Therapie bei neurologischen Erkrankungen zu einer Empfehlung durchgerungen [Simpson DM et al. Guideline Development Subcommittee of the AAN. Neurology 2016; 86: 1-9]. Danach sollte Ona-BTX A Patienten mit chronischer Migräne angeboten werden, um die Zahl kopfschmerzfreier Tage zu erhöhen und die Lebensqualität zu verbessern. Grundlage der Empfehlung sind zwei placebokontrollierte Studien, in denen es zu einem Rückgang der Kopfschmerzen pro Monat um 1,4 und 2,3 Tage kam. In beiden Studien fiel ein deutlicher Placeboeffekt auf. Eine Vergleichsstudie mit Topiramat deutete auf eine ähnliche Wirksamkeit, jedoch sieht die AAN aufgrund einer Studie keine ausreichende Evidenz, um von vergleichbarer Effektivität auszugehen.

Für episodische Migräne mit weniger als 15 Kopfschmerztagen pro Monat sehen die Experten keinen Nutzen. In zwei placebokontrollierten Studien mit 180 Tagen Dauer ließ sich unter Ona-BTX kein signifikanter Vorteil im Vergleich zu Placebo zeigen. Thomas Müller 